\title{
Essa eu vou postar: Explorando as relações entre narcisismo, uso do Instagram e a moderação da autoestima
}

\author{
This I will post: Exploring the relationship \\ between narcissism, use of Instagram and \\ moderation role of self-esteem
}

\section{Esto lo voy a publicar: Explorando las relaciones entre narcisismo, uso del Instagram y la moderación de la autoestima}

Renan P. Monteiro

ORCID ID: 0000-0002-5745-3751

Universidade Federal de Mato Grosso, Brasil

Tatiana Medeiros Costa Monteiro ORCID ID: 0000-0002-5873-5929

Centro de Estudos da Família e do Indivíduo, Brasil

Vitória Crancio Maciel

ORCID ID: 0000-0002-6285-7689

Universidade Federal de Mato Grosso, Brasil

Fábio Nasser de A. Masotti ORCID ID: 0000-0002-1054-4584 Universidade Federal de Mato Grosso, Brasil

Igor Matheus de Souza Freitas ORCID ID: 0000-0002-6592-3039 Universidade Federal de Mato Grosso, Brasil

Juarid Candido ORCID ID: 0000-0002-3229-8652 Universidade Federal de Mato Grosso, Brasil

Autor referente: renanpmonteiro@gmail.com 
Historia editorial

Recibido: 14/06/2019

Aceptado: 19/05/2020

\section{RESUMO}

O narcisismo envolve características como exibicionismo, grandiosidade e necessidade constante de ser admirado. Tais características apontam o papel fundamental que este traço de personalidade pode ter para explicar o uso do Instagram. Esta rede social parece ser um cenário propício para que os narcisistas possam se autopromover e manterem seu autoconceito grandioso e irrealístico, buscando feedback positivo por meio de curtidas, visualizações e comentários. Nesta direção, o presente estudo objetiva analisar 0 papel preditor do narcisismo em relação ao uso do Instagram, testando o papel moderador da autoestima. Participaram 207 usuários desta rede social, sendo identificado que narcisistas tendem a passar mais tempo no Instagram, postam mais fotos e stories, atribuindo muita importância a receber feedback (curtidas e visualizações). Ademais, verificou-se que a importância atribuída a receber feedbacks foi mais forte entre os narcisistas que possuem baixa autoestima, sendo que receber curtidas e visualizações pode ser uma forma de compensação para os baixos níveis nesta variável. Portanto, observa-se que 0 uso do Instagram pode ser explicado por meio do narcisismo, podendo haver interações com a autoestima, dando importantes insights para se entender o uso problemático desta rede social.

Palavras-chave: Narcisismo; Instagram; autoestima; moderação

\section{ABSTRACT}

Narcissism involves characteristics such as exhibitionism, grandiosity and a constant need to be admired. Such characteristics highlight the role that this personality trait may have in the use of Instagram. This social network seems to be a propitious setting for narcissists to self-promote and maintain their grandiose and unrealistic selfconcept by seeking positive feedback through number of likes, views, and comments. Thus, the present study aims to analyze the predictive role of narcissism in Instagram use, testing the moderating role of self-esteem. Participants were 207 users of this social network, whereby it was identified that narcissists tend to spend more time on Instagram, post more pictures and stories, giving much importance to receiving feedback (likes and views). Moreover, it was verified that the importance attributed to receiving such feedback was stronger among the narcissists with low selfesteem, such that gathering likes and views can be a way of compensating for low self-esteem. Therefore, it was observed that the use of Instagram can be explained by narcissism, and there may be interactions with self-esteem, which gives important insights to the understanding of the problematic use of this social network. 
Keywords: Narcissism; Instagram; self-esteem; moderation

\section{RESUMEN}

El narcisismo involucra características como el exhibicionismo, grandiosidad y necesidad constante de ser admirado. Dichas características apuntan el papel fundamental que este rasgo de personalidad puede tener para explicar el uso del Instagram. Esta red social parece ser un escenario propicio para que los narcisistas puedan autopromoverse y mantener su autoconcepto grandioso y irrealístico, buscando feedback positivo por medio de curtidas, visualizaciones y comentarios. En esta dirección, este estudio tiene por objetivo analizar el rol predictor del narcisismo con relación al uso del Instagram, evaluando el rol moderador de la autoestima. 207 personas usuarias de Instagram participaron del estudio, teniendo en cuenta que narcisistas pasan más tiempo en Instagram, publican más fotos y stories, atribuyendo mucha importancia a recibir feedbacks. Además, se observó que la importancia atribuida a recibir feedbacks fue más elevada entre los narcisistas con baja autoestima, siendo que recibir curtidas y visualizaciones pueden ser una forma de compensación para los bajos niveles en esta variable. Por lo tanto, se observa que el uso del Instagram puede ser explicado por medio del narcisismo, pudiendo haber interacciones con la autoestima, dando importantes claves para entender el uso problemático de esta red social.

Palavras-clave: Narcisismo; Instagram; autoestima; moderación

$\mathrm{O}$ Instagram é uma rede social onde os usuários compartilham fotos e vídeos. Criado em 2010, se expandiu rapidamente. Em 2018 contava com aproximadamente 1 bilhão de usuários, sendo 66 milhões brasileiros (Statista, 2019), ocupando o posto de quinto aplicativo mais baixado no mundo (Sensor Tower, 2019). As evidências empíricas são consistentes ao apontar que o uso problemático do Instagram pode estar relacionado a problemas como ansiedade, depressão e solidão (Yurdagül, Kircaburun, Emirtekin, Wang, \& Griffiths, 2019), além de a exposição frequente a imagens idealizadas levar os usuários a terem um decréscimo na auto avaliação de atratividade, contribuindo para o aumento do mal-estar psicológico (Sherlock \& Wagstaff, 2018). Ademais, estudar o comportamento nas redes sociais é fundamental, na medida em que os perfis em tais redes refletem os reais traços de 
personalidade das pessoas (Back et al., 2010). Não obstante, apesar de o Brasil ser o segundo país no mundo com o maior número de usuários, não encontramos estudos empíricos sobre o uso do Instagram e os seus correlatos psicológicos. Portanto, o presente estudo buscará aportar com a literatura brasileira explicando o padrão de uso desta rede social a partir de traços narcisistas da personalidade, explorando o papel moderador que a autoestima pode ter neste contexto.

\section{Narcisismo}

O Manual Diagnóstico e Estatístico dos Transtornos Mentais (DSM-V) descreve o narcisismo como um transtorno de personalidade caracterizado por um padrão consistente de grandiosidade, necessidade constante de ser admirado, acompanhado por falta de empatia e senso de direitos, tendo comportamento explorador e usando os demais em benefício próprio (APA, 2013). A abordagem da psicologia social e da personalidade tem entendido o narcisismo a partir de uma perspectiva dimensional, tendo uma variação subclínica, que compartilha aspectos com sua manifestação patológica (e.g., grandiosidade, baixa empatia, senso de direitos, autoadmiração; Jonason, Webster, Schmitt, Li, \& Crysel, 2012). Portanto, as pessoas se distribuem ao longo de um contínuo em razão da magnitude de narcisismo que apresentam.

O narcisismo normal, ou subclínico, é marcado por dominância, autoconfiança, exibicionismo e falsa modéstia (Back et al., 2013; Krizan \& Johar, 2012), sendo o Narcissistic Personality Inventory (NPI-40; Raskin \& Terry, 1988) o instrumento mais utilizado para a sua avaliação. Esta medida tem sido tratada pelos pesquisadores como um instrumento unidimensional, cobrindo uma diversidade de domínios do construto (e.g., superioridade, exibicionismo, senso de direitos, auto absorção; Raskin \& Terry, 1988). A propósito, McCain e Campbell (2018) indicam que boa parte das relações entre narcisismo e uso do Instagram foi explorada a partir da NPI-40 e sua versão reduzida, NPI-16 [NPI-40 (33\%) e NPI-16 (35\%); 68\% dos 62 estudos], o que 
indica a importância de usar tal medida neste contexto, facilitando comparações com outros estudos estimando eventuais diferenças culturais.

\section{Narcisismo e uso de redes sociais}

Considerando as características previamente listadas que demarcam um perfil narcisista, percebe-se o papel preditor que este traço pode ter para explicar o uso de redes sociais, em específico o Instagram. A propósito, as redes sociais são um espaço propício para a autopromoção (Sheldon \& Bryant, 2016), sendo que a postagem de selfies é uma atividade comum que exemplifica tal tendência (Jin \& Muqaddam, 2018). De acordo com Campbell e McCain (2018), narcisistas usam redes sociais para se autopromoverem, o que explica o fato de que narcisistas postam mais selfies, atualizam as fotos de seu perfil frequentemente, avaliando-as de forma mais atraente e passando mais tempo no Instagram (McCain et al., 2016; Moon, Lee, Lee, Choi, \& Sung, 2016; Weiser, 2015). Ademais, narcisistas costumam postar nas redes sociais tópicos sobre suas realizações pessoais e sobre a prática de exercícios (Marshall, Lefringhausen, \& Ferenczi, 2015), tirando fotos em academias ou em poses mais sensuais.

Recentemente, por meio de uma meta-análise composta por 62 amostras independentes ( $N=13.430)$, verificou-se que o narcisismo subclínico se relacionou positivamente com o tempo gasto nas redes sociais, frequência de atualizações nos status, número de amigos/seguidores e o número de selfies postadas (McCain \& Campbell, 2018). Portanto, o conjunto de evidências mostra que o Instagram pode ser encarado como um palco ideal, onde o narcisista expressará suas tendências exibicionistas e sua grandiosidade, esperando receber a devida atenção e reconhecimento que ele acha que lhe cabe (Barry \& McDougall, 2018).

De fato, ao postar uma foto, o narcisista espera um feedback, que vem a partir de comentários positivos, visualizações, curtidas e um número cada vez maior de 
seguidores. Esse feedback os ajuda em sua autoafirmação e na manutenção do autoconceito grandioso e irrealístico (Campbell \& McCain, 2018). Especificamente, narcisistas que possuem baixa autoestima tendem a postar mais selfies (March \& McBean, 2018), além de usarem filtros e hashtags e se utilizarem de comportamentos enganosos para conseguir curtidas (e.g., comprar curtidas e seguidores, melhorar a aparência em programas de edição de fotos; Dumas, Maxwell-Smith, Davis, \& Giulietti, 2017). Estes autores apontam, ainda, que essas buscas por curtidas refletem uma intensa procura por visibilidade, atenção, admiração e validação externa. Portanto, considerando a tendência para a autopromoção, narcisistas usam mais filtros, utilizam programas de edição de fotos e passam mais tempo editando suas fotos (LoweCalverley \& Grieve, 2018). Como resultado prático, esperam ter uma foto (ou vídeo) mais atrativa e que chame mais atenção dos seguidores, obtendo feedback mais positivo e, consequentemente, mantendo seu autoconceito inflado, o que pode ser uma tentativa de compensar baixos níveis de autoestima (March \& McBean, 2018). Logo, percebe-se que a profusão do uso de redes sociais tem aberto um espaço importante para os estudos psicológicos. Entretanto, a recente meta-análise conduzida por McCain e Campbell (2018) considerou majoritariamente amostras de estadunidenses. Tais autores apontam a necessidade de mais estudos em diferentes amostras e contextos culturais distintos para estimar a robustez dessas relações. Seguindo tais orientações, aliado a falta de estudos brasileiros sobre o tema, iremos explorar o papel preditor do narcisismo para o uso do Instagram, analisando o papel moderador da autoestima. Tendo em vista a literatura consultada, formularam-se as seguintes hipóteses: (a) Narcisistas passam mais tempo no Instagram; (b) Narcisistas atualizam seus perfis mais frequentemente (postam mais fotos e stories); (c) Narcisistas passam mais tempo editando suas fotos e; (d) Narcisistas com baixa autoestima dão mais importância a receber feedback no Instagram (curtidas e visualizações). 


\section{Método}

\section{Participantes}

Tratou-se de uma amostra de conveniência (não-probabilística) composta por 207 pessoas que indicaram usar a rede social Instagram. Os participantes tinham idades variando entre 18 e 62 anos $\left(M_{\text {idade }}=27,08 ; D P_{\text {idade }}=10,41\right)$. A maioria declarou ser do sexo feminino $(65,7 \%)$, solteiro $(69,1 \%)$, de classe social média $(44 \%)$ e com ensino superior incompleto (43\%).

\section{Instrumentos}

Narcissistic Personality Inventory (NPI-40): Medida elaborada por Raskin e Terry (1988), sendo do tipo escolha forçada, essa é a medida mais usada para avaliar o narcisismo subclínico. Cada item é representado por um par de afirmações, cabendo à pessoa escolher a frase que melhor a descreve. Uma frase descreve uma resposta narcisista "Usualmente, tento me exibir quando tenho a oportunidade" enquanto a outra não "Tento não ser exibido". Na presente ocasião, observou-se um coeficiente de consistência interna adequado $(\alpha=0,79)$.

Escala de Autoestima de Rosenberg (EAR): Desenvolvida por Rosenberg (1965), esta medida é formada por 10 itens, sendo os participantes orientados a indicar a sua concordância (1 - Discordo Totalmente; 7 - Concordo Totalmente) a itens como "Sou capaz de fazer tudo tão bem como as outras pessoas" e "Eu acho que tenho muitas boas qualidades". Na presente ocasião, observou-se um coeficiente de consistência interna adequado $(\alpha=0,89)$.

Uso do Instagram: Para definir o padrão de uso deste aplicativo perguntou-se aos usuários o tempo gasto diariamente nesta rede social (1 - Menos de 15 minutos; 4 Mais de 60 minutos), tempo gasto para editar uma foto antes de postá-la (1 - Menos de um minuto; 7 - Mais de dez minutos), frequência com que posta stories (1 - Nunca; 10 - Sempre) e o quão importante é receber curtidas nas fotos e visualizações nos 
stories, ambos respondidos em escala de 10 pontos (1 - Nada importante; 10 Extremamente importante). Por fim, os participantes foram indagados sobre o número aproximado de fotos e selfies que possuem no Instagram, sendo esta uma pergunta aberta.

\section{Procedimento}

Os dados foram coletados por meio online, sendo o questionário criado na plataforma Google Formulários e o link compartilhado nas redes sociais (e.g., Facebook, Whatsapp) a partir do procedimento bola de neve. Especificamente, solicitamos aos respondentes que, ao término do preenchimento do questionário, divulgassem o link da pesquisa entre seus contatos, fazendo o mesmo apelo. Antes de prosseguir para o preenchimento dos questionários, demandava-se que o participante lesse o Termo de Consentimento Livre e Esclarecido (TCLE), com informações sobre os objetivos da pesquisa, ressaltando o caráter anônimo e voluntário da participação, indicando que poderiam desistir da colaboração a qualquer momento. Os participantes que concordaram com o TCLE, prosseguiram para o preenchimento dos instrumentos. Portanto, os princípios éticos que orientam pesquisas com seres humanos foram seguidos, de acordo com a Resolução 510/2016 do Conselho Nacional de Saúde.

\section{Análise de Dados}

Os dados foram analisados por meio do pacote estatístico IBM SPSS. Especificamente, foram calculadas estatísticas descritivas com o objetivo de caracterização da amostra (e.g., média e desvio padrão), coeficiente alfa de Cronbach, buscando avaliar a fidedignidade da NPI-40 e EAR, análise de correlação de Pearson para conhecer em que medida e direção as variáveis estão relacionadas e análise de regressão hierárquica, para verificar o papel moderador da autoestima na relação narcisismo e uso do Instagram. Por fim, utilizou-se o ModGraph-I (Jose, 2013) para 
gerar as figuras da moderação e calcular os simple slopes, que indicam para quais grupos a interação é estatisticamente significativa.

\section{Resultados}

Inicialmente, calcularam-se análises de correlação de Pearson para conhecer o padrão de correlações entre as variáveis. Especificamente, observou-se que narcisistas passam mais tempo no Instagram $(r=0,19 ; p<0,01)$, postam mais fotos $(r$ $=0,15 ; p<0,05)$ e stories $(r=0,26 ; p<0,01)$, atribuindo mais importância a obtenção de curtidas $(r=0,34 ; p<0,01)$ e visualizações $(r=0,30 ; p<0,01)$, além de passarem mais tempo editando as suas fotos $(r=0,21 ; p<0,01)$. No entanto, as correlações entre a frequência de selfies postadas e traços narcisistas não foram significativas. Todas as correlações são especificadas na Tabela 1.

\section{Tabela 1}

Correlação entre narcisismo, autoestima e uso do Instagram

\section{NA}

AE $0,24^{* *}$

TI $0,19^{* *}-0,18^{* *}$

FS $0,26^{* *} \quad-0,03 \quad 0,43^{* *}$

NF $\quad 0,15^{*} \quad-0,11 \quad 0,38^{* *} \quad 0,39^{* *}$

NS $\quad 0,09$

$-0,11^{*}$

$0,19^{* *}$

$0,22^{* *}$

$0,75^{* *}$

IC $0,34^{* *}$

$-0,12^{*}$

$0,30^{* *}$

$0,39 * * \quad 0,20$ **

$0,15^{*}$

IV $0,30^{* *}$

$-0,10$

$0,35^{* *}$

$0,51^{* *} \quad 0,22^{* *}$

$0,17^{* *} \quad 0,67^{* *}$

TE $0,21^{* *} \quad-0,03$

$0,29^{* \star}$

$0,30^{* *}$

0,10

0,02

$0,32^{* *} \quad 0,22^{* *}$

NA AE TI FS NF NS IC IV

Nota: ${ }^{*} p<0,05,{ }^{* *} p<0,001$ (teste uni-caudal). Identificação das variáveis: NA = Narcisismo, AE = Autoestima, $\mathrm{TI}=$ Tempo gasto no Instagram, $\mathrm{FS}=$ Frequência com que posta stories, $\mathrm{NF}=$ Número de fotos, NS = Número de selfies, IC = Importância atribuída a curtidas, IV = Importância atribuída a visualizações, e TE = Tempo editando fotos. 
O passo seguinte foi realizar análises de regressão hierárquica (método enter) buscando conhecer o papel moderador da autoestima na relação entre narcisismo e as variáveis relativas ao uso da rede social em questão (frequência de uso, número de postagens, importância atribuída a curtidas e visualizações e tempo gasto editando fotos). Padronizou-se $(z)$ as variáveis narcisismo e autoestima, multiplicando-as e computando o termo de interação. No primeiro passo das cinco análises de regressão, entrou-se com a idade e o sexo dos participantes, no segundo passo foram introduzidos os valores padronizados do narcisismo e autoestima, sendo o termo de interação incluído no terceiro passo da análise. Considerando as relações testadas, o termo de interação predisse duas variáveis: a importância atribuída a curtidas e visualizações, configurando o papel moderador da autoestima (Tabela 2).

Tabela 2

Regressões hierárquicas

\begin{tabular}{lllll}
\hline \multicolumn{5}{c}{ Variável consequente: Importância de curtidas } \\
& $\mathrm{b}$ & $\beta$ & $\mathrm{R}^{2}$ & $\Delta \mathrm{R}^{2}$ \\
\hline Passo 1 & & & 0,024 & 0,024 \\
Idade & $-0,04$ & $-0,14^{*}$ & & \\
Sexo & $-0,39$ & $-0,07$ & & \\
Passo 2 & & & 0,163 & $0,139^{* *}$ \\
Idade & $-0,005$ & $-0,02$ & & \\
Sexo & $-0,25$ & $-0,04$ & & \\
Narcisismo & 1,06 & $0,39^{\star *}$ & & \\
Autoestima & $-0,54$ & $-0,20^{\star *}$ & & \\
Passo 3 & & & 0,191 & $0,028^{*}$ \\
Idade & $-0,01$ & $-0,03$ & & \\
Sexo & $-0,25$ & $-0,04$ & & \\
Narcisismo & 1,09 & $0,40^{* *}$ & & \\
Autoestima & $-0,53$ & $-0,19^{* *}$ & \\
Interação & $-0,47$ & $-0,17^{* *}$ & \\
\hline
\end{tabular}

(Continuação) 
Tabela 3 Regressões hierárquicas (Continuação)

\begin{tabular}{lllll}
\hline \multicolumn{5}{c}{ Variável consequente: Importância de visualizações } \\
& $\mathrm{B}$ & $\mathrm{B}$ & $\mathrm{R}^{2}$ & $\Delta \mathrm{R}^{2}$ \\
\hline Passo 1 & & & 0,028 & 0,028 \\
Idade & $-0,05$ & $-0,16^{*}$ & & \\
Sexo & 0,04 & 0,01 & & \\
Passo 2 & & & 0,131 & $0,103^{* *}$ \\
Idade & $-0,02$ & $-0,06$ & & \\
Sexo & 0,17 & 0,03 & & \\
Narcisismo & 0,99 & $0,34^{* *}$ & & \\
Autoestima & $-0,48$ & $-0,16^{*}$ & & \\
Passo 3 & & & 0,187 & $0,056^{* *}$ \\
Idade & $-0,02$ & $-0,08$ & & \\
Sexo & 0,17 & 0,03 & & \\
Narcisismo & 1,03 & $0,35^{* *}$ & & \\
Autoestima & $-0,46$ & $-0,16^{*}$ & & \\
Interação & $-0,72$ & $-0,24^{* *}$ & & \\
\hline
\end{tabular}

Especificamente para a predição da importância atribuída a curtidas, observou-se que no primeiro passo da análise, idade e sexo explicaram $2,4 \%$ da variância $(p<0,01)$. Incluindo os valores padronizados do narcisismo e autoestima, a variância explicada passou para $16,3 \%$, sendo esse incremento significativo $(p<0,01)$. Ademais, no terceiro passo, foi incluído o termo de interação, sendo que a variância explicada passou para 19,1\%, resultando em um aumento significativo na explicação $(p<0,01)$. No último passo da análise, narcisismo $(\beta=0,40 ; p<0,01)$, autoestima $(\beta=-0,19 ; p<$ $0,01)$ e o termo de interação $(\beta=-0,17 ; p<0,01)$ predisseram a importância atribuída a receber curtidas. Por meio do Mod-Graph I (Jose, 2013) observou-se que as relações entre narcisismo e valorização de curtidas são mais fortes entre as pessoas que possuem baixa (simple slope $=1,56 ; t=5,92 ; p<0,001$ ) e média autoestima (simple slope $=1,09 ; t=5,92 ; p<0,001$ ), sendo mais fraca entre aqueles com elevada 
autoestima (simple slope $=0,61 ; t=2,46 ; p<0,05$ ). O gráfico de moderação pode ser observado na Figura 1.
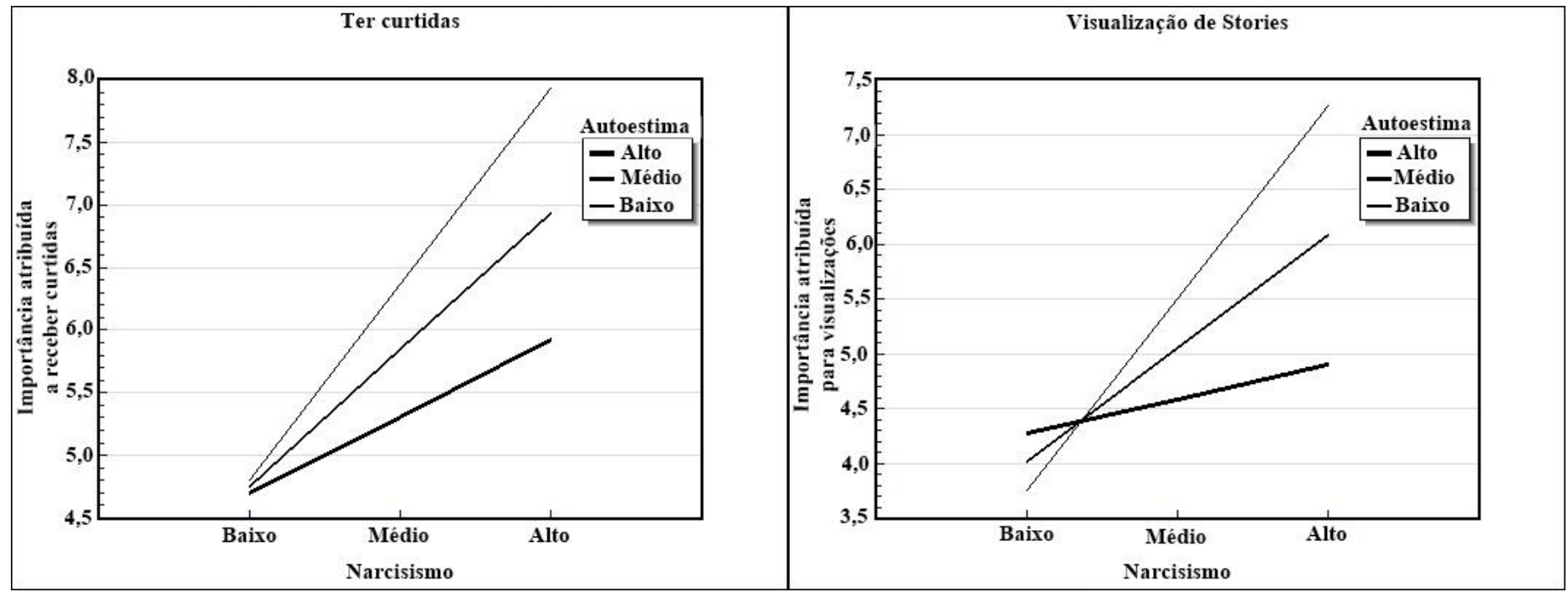

Figura 1. Moderação da autoestima na relação entre narcisismo e importância de feedback

Por outro lado, para a predição da importância atribuída a receber visualizações, observou-se que no primeiro passo da análise, idade e sexo explicaram $2,8 \%$ da variância $(p<0,01)$. Adicionando os valores padronizados do narcisismo e da autoestima, a explicação sobe, de forma significativa $(p<0,01)$, para $13,1 \%$. Com a inserção do termo de interação no último passo, a explicação passou para $18,7 \%$, sendo esse aumento significativo $(p<0,01)$. No último passo da análise, narcisismo ( $\beta$ $=0,35 ; p<0,01)$, autoestima $(\beta=-0,17 ; p<0,05)$ e o termo de interação $(\beta=-0,24 ; p$ $<0,01)$ predisseram a importância atribuída para receber visualizações. Por meio do Mod-Graph I (Jose, 2013), observou-se que as relações entre narcisismo e importância atribuída a receber visualizações de stories são mais fortes entre as pessoas que possuem baixa (simple slope $=1,75 ; t=6,15 ; p<0,001$ ) e média autoestima (simple slope $=1,03 ; t=5,20 ; p<0,001$ ). Entre aquelas com elevada autoestima, não há relação entre narcisismo e importância atribuída a ter visualizações 
(simple slope $=0,31 ; t=1,16 ; p>0,05)$. gráfico de moderação pode ser observado na Figura 1.

\section{Discussão}

O Instagram é uma das redes sociais que mais cresce atualmente, sendo que o Brasil ocupa a segunda posição mundial em número de usuários. Apesar do número crescente de estudos que exploram os preditores do uso desta rede social e suas potenciais consequências, as evidências em contexto brasileiro são inexistentes. Nesta direção, o presente estudo contribuiu com a literatura nacional indicando o importante papel preditor do narcisismo para o uso do Instagram.

Especificamente, verificamos que os narcisistas usam mais o Instagram, postando uma quantidade maior de fotos e stories, passando mais tempo editando-as, além de darem muita importância para feedbacks (curtidas e visualizações), confirmando, assim, as hipóteses 1, 2 e 3. Tais resultados se somam aos reportados previamente na literatura (Dumas et al., 2017; McCain \& Campbell, 2018; McCain et al., 2016; Moon et al., 2016), indicando que pessoas com traços narcisistas usam as redes sociais com a finalidade de autopromoção, sendo o Instagram um palco propício para a exibição de tais tendências (Sheldon \& Bryant, 2016).

Não obstante, para receberem um feedback positivo, e reforçarem seu ego frágil, narcisistas passam mais tempo editando suas fotos (Lowe-Calverley \& Grieve, 2018). De fato, no presente estudo, verificou-se relação positiva entre narcisismo e tempo gasto na edição de fotos, o que vai na mesma direção de estudos prévios que verificaram que pessoas com este traço de personalidade usam mais filtros, melhoram a aparência em programas de edição de fotos e passam mais tempo editando-as (Dumas et al., 2017; Lowe-Calverley \& Grieve, 2018). Aparentar uma imagem melhor se relaciona a um feedback mais positivo que, por sua vez, ajuda os narcisistas em sua autoafirmação e manutenção do autoconceito grandioso e irrealístico (Campbell \& 
McCain, 2018). A propósito, confirmamos a hipótese 4, com evidências que reforçam esse mecanismo. A busca por popularidade e destaque nas redes sociais leva a um uso cada vez mais intenso (refletido na preocupação em obter curtidas e visualizações como uma forma de aprovação social), e isso seria uma forma dos narcisistas compensarem a baixa autoestima (March \& McBean, 2018). É importante ressaltar que em razão desse uso intenso, narcisistas com baixa autoestima podem ser um grupo de risco para terem problemas de depressão, ansiedade e solidão em razão do uso problemático do Instagram (Yurdagül et al., 2019).

Os resultados apresentados, apesar de convergirem com a literatura, devem ser analisados com ressalvas. Por exemplo, tratou-se de uma amostra de conveniência, impossibilitando generalizações para além da amostra utilizada. Outro problema se refere a forma como se quantificou o número de fotos, selfies postadas e stories. Os dois primeiros por meio de pergunta aberta e o último em escala Likert. Um possível viés inerente é que as pessoas podem ter dado informações que subestimam ou superestimam o número de postagens. A propósito, estudos têm usado diferentes métodos, a exemplo de escalas do tipo Likert que avaliam a frequência de selfies postadas (1 - Raramente; 7 - Várias vezes por dia; Singh, Farley, \& Donahue, 2018) ou mesmo solicitar que o participante indique o nome do seu perfil no Instagram para que o pesquisador o analise por um período específico de tempo (Barry, Reiter, Anderson, Schoessler, \& Sidoti, 2019), sendo que essa última estratégia pode ser usada em possibilidades futuras.

Contudo, apesar de algumas limitações, no geral os resultados convergem com as evidências prévias descritas na literatura (McCain \& Campbell, 2018; Moon et al., 2016; Weiser, 2015). A propósito, atendendo a sugestões da literatura para a ampliação dos estudos para diferentes contextos socioculturais (McCain \& Campbell, 2018), observou-se que o narcisismo apresenta um papel consistente para explicar o uso do Instagram no contexto brasileiro. Não obstante, ainda há muito a ser explorado. 
Por exemplo, predizer o tipo de conteúdo postado por narcisistas nas redes sociais ou mesmo desenvolver um estudo qualitativo buscando apreender as motivações para fazer uso de tal rede social. Ademais, em possibilidades futuras, é primordial ampliar a lista de preditores do uso deste aplicativo, possibilitando a identificação de perfis psicológicos que tem mais risco para um uso problemático e disfuncional. Logo, percebe-se que as possibilidades são diversas, indicando que há um campo fértil para a condução de estudos psicológicos, sobretudo se considerarmos que o Brasil é um dos países com maior número de usuários de redes sociais.

\section{Referências}

American Psychiatric Association. (2013). Diagnostic and statistical manual of mental disorders (DSM-V) (5 $\left.5^{\mathrm{a}} e d.\right)$. Washington, DC: American Psychiatric Association.

Back, M. D., Küfner, A. C., Dufner, M., Gerlach, T. M., Rauthmann, J. F., \& Denissen, J. J. (2013). Narcissistic admiration and rivalry: Disentangling the bright and dark sides of narcissism. Journal of Personality and Social Psychology, 105, 1013-1037. https://doi.org/10.1037/a0034431

Back, M. D., Stopfer, J. M., Vazire, S., Gaddis, S., Schmukle, S. C., Egloff, B., \& Gosling, S. D. (2010). Facebook profiles reflect actual personality, not selfidealization. Psychological $\quad$ Science, $21, \quad 372-374$. https://doi.org/10.1177/0956797609360756

Barry, C. T., \& McDougall, K. H. (2018). Social Media: Platform or Catalyst for Narcissism? In A. D. Hermann, A. D. Brunell, \& J. D. Foster (Eds.), Handbook of Trait Narcissism: Key Advances, Research Methods, and Controversies (pp. 435-441). Cham, Switzerland: Springer.

Barry, C. T., Reiter, S. R., Anderson, A. C., Schoessler, M. L., \& Sidoti, C. L. (2019). "Let me take another selfie": Further examination of the relation between 
narcissism, self-perception, and Instagram posts. Psychology of Popular Media Culture, 8, 22-33. https://doi.org/10.1037/ppm0000155

Campbell, W. K., \& McCain, J. (2018). Theoretical Perspectives on Narcissism and Social Media: The Big (and Beautiful) Picture. In A. D. Hermann, A. D. Brunell, \& J. D. Foster (Eds.), Handbook of Trait Narcissism: Key Advances, Research Methods, and Controversies (pp. 443-453). Cham, Switzerland: Springer.

Dumas, T. M., Maxwell-Smith, M., Davis, J. P., \& Giulietti, P. A. (2017). Lying or longing for likes? Narcissism, peer belonging, loneliness and normative versus deceptive like-seeking on Instagram in emerging adulthood. Computers in Human Behavior, 71, 1-10. https://doi.org/10.1016/j.chb.2017.01.037

Jin, S. V., \& Muqaddam, A. (2018). "Narcissism 2.0! Would narcissists follow fellow narcissists on Instagram?" the mediating effects of narcissists personality similarity and envy, and the moderating effects of popularity. Computers in Human Behavior, 81, 31-41. https://doi.org/10.1016/j.chb.2017.11.042

Jose, P.E. (2013). ModGraph-I: A programme to compute cell means for the graphical display of moderational analyses: The internet version, Version 3.0. Victoria University of Wellington, Wellington, New Zealand. Retirado de https://psychology.victoria.ac.nz/modgraph/

Jonason, P. K., Webster, G. D., Schmitt, D. P., Li, N. P., \& Crysel, L. (2012). The antihero in popular culture: Life history theory and the dark triad personality traits. Review of General Psychology, 16, 192-199. https://doi.org/10.1037/a0027914

Krizan, Z., \& Johar, O. (2012). Envy divides the two faces of narcissism. Journal of Personality, 80, 1415-1451. https://doi.org/10.1111/j.1467-6494.2012.00767.x

Lowe-Calverley, E., \& Grieve, R. (2018). Selfie love: Predictors of image editing intentions on Facebook. Telematics and Informatics, 35, 186-194. https://doi.org/10.1016/j.tele.2017.10.011 
March, E., \& McBean, T. (2018). New evidence shows self-esteem moderates the relationship between narcissism and selfies. Personality and Individual Differences, 130, 107-111. https://doi.org/10.1016/j.paid.2018.03.053

Marshall, T. C., Lefringhausen, K., \& Ferenczi, N. (2015). The Big Five, self-esteem, and narcissism as predictors of the topics people write about in Facebook status updates. Personality and Individual Differences, 85, 35-40. https://doi.org/10.1016/j.paid.2015.04.039

McCain, J. L., Borg, Z. G., Rothenberg, A. H., Churillo, K. M., Weiler, P., \& Campbell, W. K. (2016). Personality and selfies: Narcissism and the Dark Triad. Computers in Human Behavior, 64, 126-133. https://doi.org/10.1016/j.chb.2016.06.050

McCain, J. L., \& Campbell, W. K. (2018). Narcissism and social media use: A metaanalytic review. Psychology of Popular Media Culture, 7, 308-327. http://dx.doi.org/10.1037/ppm0000137

Moon, J. H., Lee, E., Lee, J. A., Choi, T. R., \& Sung, Y. (2016). The role of narcissism in self-promotion on Instagram. Personality and Individual Differences, 101, 2225. https://doi.org/10.1016/j.paid.2016.05.042

Raskin, R., \& Terry, H. (1988). A principal-components analysis of the Narcissistic Personality Inventory and further evidence of its construct validity. Journal of Personality and Social Psychology, 54, 890-902. Recuperado de http://www.sakkyndig.com/psykologi/artvit/raskin1988.pdf

Rosenberg, M. (1965). Society and the adolescent self-image. Princeton: Princeton University Press.

Sensor Tower (2019). Top apps worldwide for Q1 2019. Recuperado de https://sensortower.com/blog/top-apps-worldwide-q1-2019-downloads 
Sheldon, P., \& Bryant, K. (2016). Instagram: Motives for its use and relationship to narcissism and contextual age. Computers in Human Behavior, 58, 89-97. https://doi.org/10.1016/j.chb.2015.12.059

Sherlock, M., \& Wagstaff, D. L. (2018). Exploring the relationship between frequency of Instagram use, exposure to idealized images, and psychological well-being in women. Psychology of Popular Media Culture. https://dx.doi.org/10.1037/ppm0000182

Singh, S., Farley, S. D., \& Donahue, J. J. (2018). Grandiosity on display: Social media behaviors and dimensions of narcissism. Personality and Individual Differences, 134, 308-313. https://dx.doi.org/10.1016/j.paid.2018.06.039

Statista. (2019). Leading countries based on number of Instagram users as of April 2019 (in millions). Recuperado de https://www.statista.com/statistics/578364/countries-with-most-instagram-users/

Weiser, E. B. (2015). \# Me: Narcissism and its facets as predictors of selfie-posting frequency. Personality and Individual Differences, 86, 477-481. https://doi.org/10.1016/j.paid.2015.07.007

Yurdagül, C., Kircaburun, K., Emirtekin, E., Wang, P., \& Griffiths, M. D. (2019). Psychopathological consequences related to problematic Instagram use among adolescents: The mediating role of body image dissatisfaction and moderating role of gender. International Journal of Mental Health and Addiction, 1-13. https://doi.org/10.1007/s11469-019-00071-8

\section{Declaração do contributo dos autores}

RPM e TMCM contribuíram com o desenho do estudo, análise dos dados e na escrita do manuscrito, VCM, FNAM, IMSF e JC contribuíram com a coleta e análise dos dados. Todos os autores discutiram os resultados e contribuíram para a versão final do manuscrito. 


\section{Editor de sección}

El editor de sección de este artículo fue Victor Ortuño.

ORCID ID: 0000-0002-9523-0874

Formato de citación

Monteiro, R.P., Monteiro, T.M.C., Maciel, V.C., Masotti, F.N.A., Freitas, I.S. \& Candido, J. (2020). Essa eu vou postar: Explorando as relações entre narcisismo, uso do Instagram e a moderação da autoestima. Psicología, Conocimiento y Sociedad, 10(2), 55-73. doi: http://dx.doi.org/10.26864/PCS.v10.n2.3 